\title{
EXPOSIÇÃO AO RUÍDO DO TRANSPORTE RODOVIÁRIO EM BRASÍLIA, BRASIL
}

\author{
EXPOSURE TO ROAD TRAFFIC NOISE IN BRASÍLIA, BRAZIL
}

\author{
(D) Sérgio Luiz Garavelli1 \\ (D)Armando de Mendonça Maroja² \\ DEdson Benício de Carvalho \\ Júnior ${ }^{3}$ \\ (D) Cleber Alves da Costa 4 \\ ${ }^{1}$ Centro Universitário de Brasília, Brasília, \\ DF, Brasil, sergio.garavelli@ceub.edu.br \\ ${ }^{2}$ Universidade de Brasília, Planaltina, \\ Distrito Federal, amaroja@unb.br \\ ${ }^{3}$ Universidade Católica de Brasília, Águas \\ Clara, DF, edsonbenicio@ucb.br \\ ${ }^{4}$ Universidade Católica de Brasília, Águas \\ Clara, DF, cleberalves@ucb.br
}

\section{Contribuição dos autores:}

SLG: conceituação, curadoria de dados, análise formal, aquisição de

financiamento, investigação, metodologia, administração de projetos, programas, supervisão, validação, visualização, escrita - rascunho original. escrita - revisão e edição. AMM: conceituação, curadoria de dados, análise formal, aquisição de

financiamento, investigação, metodologia, administração de projetos, programas, supervisão, validação, visualização, escrita - rascunho original. escrita - revisão e edição. EBCJR: curadoria de dados, análise formal, investigação, validação, escrita rascunho original. CAC: curadoria de dados, análise formal, investigação, validação, visualização, escrita - rascunho original.

Fomento: Organização das Nações Unidas para a Educação, a Ciência e a Cultura. Conselho Nacional de Desenvolvimento Científico e Tecnológico

Declaração de conflito: nada foi declarado.

Editor Responsável:

Vanessa Gomes da Silva $\mathbb{D}$

\begin{abstract}
Resumo
A contaminação acústica é um dos principais problemas ambientais nos grandes centros urbanos gerados pelo tráfego rodoviário. Os mapas acústicos são ferramentas importantes para a gestão do ruído nestes locais. O objetivo principal deste artigo é apresentar os resultados da avaliação do impacto acústico devido ao tráfego rodoviário sobre a população de Brasília. $O$ trabalho também apresenta uma metodologia alternativa para aquisição dos dados do tráfego, necessários para elaboração dos mapas acústicos. A velocidade, o fluxo e a composição do tráfego foram obtidos a partir dos dados fornecidos pelos controladores eletrônicos de velocidade, comuns na maioria das cidades e complementados com filmagens e medições acústicas in situ. Na elaboração dos mapas acústicos foi utilizado software de simulação e seguidas as recomendações da Comunidade Europeia. A partir da análise dos mapas, foram estimados o incomodo devido ao tráfego rodoviário e o número de pessoas sujeitas à distúrbios no sono. Os resultados indicam que a metodologia alternativa proposta é adequada e pode ser utilizada em outras cidades, diminuindo os custos para elaboração dos mapas, que são elevados. Os resultados mostram ainda que apesar do trânsito intenso na cidade, os percentuais de pessoas altamente incomodadas e sujeitas a distúrbios no sono é relativamente pequeno. A estrutura hierárquica, a forma das vias e o modelo de Superquadras utilizados em Brasília, contribuem para a melhoria das condições de conforto acústico, ou seja, os resultados deste artigo mostram uma influência positiva da concepção urbanística nas condições de conforto acústico da cidade.
\end{abstract}

Palavras-chave: Mapa acústico. Incômodo. Transporte rodoviário. Poluição sonora. Saúde pública.

\begin{abstract}
Acoustic contamination is one of the main environmental problems in large urban centres generated by road traffic. Noise maps are important tools for noise management in these locations. The main objective is to present the results of the evaluation of the acoustic impact due to road traffic on the population of Brasília. The article also presents an alternative methodology for acquiring traffic data necessary for the elaboration of noise maps. The speed, flow and composition of traffic were obtained from data provided by electronic speed controllers, common in most cities and complemented with filming and acoustic measurements in situ. In the elaboration of the noise maps, environmental simulation software was used, and the recommendations of the European Community were followed. From the analysis of the maps, annoyance due to road traffic and the number of people subject to sleep disturbances were estimated. The results indicate that the proposed alternative methodology is adequate and can be used in other cities, reducing the high costs for preparing the maps. The results also show that despite the heavy traffic in the city, the percentage of people who are highly annoyed and subject to sleep disorders is relatively small. The hierarchical structure, the shape of the roads and the Superquadras model used in Brasília contribute to the improvement of the conditions of acoustic comfort. Therefore, results show a positive influence of urban design on the city's acoustic comfort conditions.
\end{abstract}

Keywords: Noise Map. Annoyance. Road traffic. Noise pollution. Public health

How to cite this article:

GARAVELLI, S. L.; MAROJA, A. de M.; CARVALHO JÚNIOR, E. B. de; COSTA, C. A. da. Exposição ao ruído do transporte rodoviário em Brasília, Brasil. PARC Pesq. em Arquit. e Constr., Campinas, SP, v. 12, p. e021026, 2021. DOI:

http://dx.doi.org/10.20396/parc.v12i00.8658514 


\section{Introdução}

O aumento populacional nas grandes cidades é um fenômeno mundial que vem ocorrendo desde as últimas décadas do século passado. Atualmente, cerca de $50 \%$ da população vive em centros urbanos, estima-se que em 2050 quase $70 \%$ residirá nas cidades. No Brasil, $85 \%$ da população vive em áreas urbanas. A concentração de pessoas nas cidades propicia uma maneira de fornecer mais serviços à população com custo menor, porém a necessidade de mobilidade traz como consequência o agravamento de problemas ambientais. Atualmente, as cidades são responsáveis por $70 \%$ das emissões de gases de efeito estufa e consomem $75 \%$ da energia e dos recursos do mundo (CODEPLAN, 2018).

O tráfego veicular é um grande gerador de poluição ambiental (KING; MURPHY; RICE, 2011; CAl et al., 2015; EEA, 2014), principalmente a sonora e a do ar (DE VOS; VAN BEEK, 2011), provoca impactos na economia (D'ALESSANDRO; SCHIAVONI, 2015) causando a depreciação do valor de imóveis (SEO; GOLUB; KUBY, 2014). Na saúde da população, aumenta o risco de doenças cardíacas, altera os níveis de colesterol e potencializa outras doenças, como acidente vascular cerebral, obesidade e diabetes (SØRENSEN et al., 2015; WHO, 2018).

Brasília, capital do Brasil, apesar de planejada e jovem, sofre com problemas relacionados a mobilidade urbana. Conforme pesquisa distrital por amostra de domicílios (CODEPLAN, 2018), a região central concentra $41 \%$ dos postos de trabalho, em empresas públicas, privadas e órgãos do governo Federal e Distrital. A pesquisa mostra que no deslocamento para o trabalho, $38 \%$ utilizam ônibus e $47 \%$ automóvel de pequeno porte. Assim, diariamente centenas de milhares de pessoas se dirigem ao centro da cidade em seus veículos automotores deixando em seu caminho um rastro de poluição sonora e do ar. Poluição esta que atinge de forma negativa os habitantes das margens das principais vias que atravessam a cidade.

Este artigo tem como objetivo principal apresentar os resultados da avaliação do impacto acústico do tráfego rodoviário sobre a população do Plano Piloto de Brasília, que é a primeira Região Administrativa do Distrito Federal (DF). Apresenta também uma metodologia alternativa para a aquisição dos dados referentes ao trânsito, composição do fluxo e velocidade média, dados necessários para a elaboração dos mapas de ruído.

O plano urbanístico da capital, conhecido como Plano Piloto, foi elaborado pelo urbanista Lúcio Costa, que, aproveitando o relevo da região, adequou-o ao projeto do Lago Paranoá, concebido em 1893 pela Missão Cruls (COSTA, 2011). Uma das características marcantes de Brasília são as Superquadras, que constituem a principal área residencial, possuem um contorno de vegetação, faixa verde, densamente arborizado. Segundo Lúcio Costa, "A disposição dos edifícios é de forma livre e variada, tomando o cuidado de respeitar apenas dois princípios; o gabarito máximo, talvez seis pavimentos e pilotis, e a separação do tráfego de pedestres e veículos" (Instituto Do Patrimônio Histórico e Artístico Nacional - IPHAN, 2015).

O comércio está localizado entre as quadras, sendo que cada uma possui um único acesso, o que garante um tráfego menor e com velocidade reduzida, em função da sua conformação, conforme IPHAN (2015, p.10):

A Superquadra é a mais importante contribuição de Brasília à história do urbanismo mundial. Lucio Costa, ao romper com a estrutura do quarteirão convencional, abrindo-o e transformandoo em um amplo bosque entremeado por blocos residenciais multifamiliares, de até seis pavimentos em pilotis livres, liberando o chão para uso público indistinto, concebeu uma nova maneira 
de morar em área urbana, estruturada no que ele denominou de escala residencial ou cotidiana.

$\mathrm{Na}$ atualidade, a quantificação do impacto do ruído sobre a população é uma preocupação em todo o mundo. Murphy, King e Rice (2009) estimaram a população exposta ao ruído do transporte em Dublin na Irlanda, concluíram que medidas de gestão do tráfego têm o potencial de reduzir significativamente o nível de exposição ao ruído. Seong et al. (2011), em Atlanta nos EUA, destacam a importância dos mapas acústicos que apresentem a distribuição espacial de exposição ao ruído de modo a revelar onde começar e/ou concentrar os programas de controle e redução do ruído.

Ko et al. (2011), determinaram os NPS (níveis de pressão sonora) devido ao transporte rodoviário e ferroviário, avaliaram a população exposta no período diurno e noturno em Youngdeungpo-gu, distrito da cidade de Seul, na Coréia do Sul. Os resultados da pesquisa mostram que no período noturno $80 \%$ da população está exposta a NPS acima de $40 \mathrm{~dB}$.

No Brasil, Guedes e Bertoli (2014) utilizaram mapas acústicos para avaliar cenários atuais e futuros do ruído devido ao tráfego veicular em uma avenida da cidade de Aracaju. Bunn e Zannin (2016) avaliaram medidas de mitigação para diminuição do impacto acústico devido a implantação de uma ferrovia sobre áreas sensíveis ao ruído, tais como hospitais e escolas na cidade de Curitiba. Em pesquisa realizada em São Paulo, Paiva, Cardoso e Zannin (2019) avaliaram a exposição da população adulta ao ruído do tráfego rodoviário, segundo o estudo, $48 \%$ da amostra relatou incômodo relacionado ao ruído.

Desde 2007 , os estados membros da Comunidade Europeia estão obrigados a produzir mapas estratégicos de ruído para as principais cidades, com atualização a cada cinco anos (KEPHALOPOULOS et al., 2014). A Directiva 2002/49/EC (DIRECTIVA, 2002) prevê a quantificação do ruído ambiental na fachada das edificações, que permite o cálculo da população exposta, determinar os percentuais de pessoas altamente incomodadas e com o sono altamente perturbado em função da exposição ao ruído dos transportes.

No Brasil, a legislação ainda é incipiente, somente para a cidade de São Paulo está previsto em lei a elaboração dos mapas estratégicos de ruído (SÃo PAULO, 2016). Em Brasília, as normas gerais sobre o controle da poluição sonora e os limites máximos de intensidade da emissão de sons são regulamentadas pela Lei Distrital 4.092/2008 (DISTRITO FEDERAL, 2008), que não prevê a elaboração dos mapas de ruído e não utiliza os parâmetros $L_{d e n}$ e $L_{n}$ como descritores acústicos. Destaca-se que $L_{d e n}$ e $L_{n}$ caracterizam respectivamente a exposição relativa ao ruído ambiental médio nas $24 \mathrm{~h}$ do dia (dia-entardecer-noite) e no período noturno. A definição destes parâmetros é realizada na seção a seguir.

O desenvolvimento de computadores e softwares facilitou o processo de simulação para a elaboração dos mapas acústicos, porém a obtenção dos dados necessários para alimentar os softwares ainda são dispendiosos. Neste artigo, é apresentada uma metodologia alternativa para aquisição dos dados para a elaboração de mapas acústicos. O processo é baseado nos dados dos CEV (controladores eletrônicos de velocidades), cuja malha em Brasília conta com quase uma centena de aparelhos que registram diariamente o tráfego dos veículos nas principais vias da cidade.

\section{Fundamentação}

Os mapas acústicos são ferramentas importantes para a gestão do ruído ambiental (TSAI; LIN; CHEN, 2009), sendo uma representação gráfica da distribuição espacial do ruído em uma região. Nos mapas, os NPS representativos do ruído são apresentados na 
forma de linhas de contorno ou isofônicas, caracterizando níveis iguais de exposição ao ruído.

Para modelagem são necessários os dados cartográficos referentes as curvas de nível, localização dos edifícios e do eixo das vias, além da caracterização das fontes de ruído: o fluxo de veículos por hora nas $24 \mathrm{~h}$ do dia, a composição do tráfego e a velocidade, entre outras. Nem sempre essas informações estão disponíveis, em muitos casos quando existem são limitadas, havendo necessidade de simplificações como agrupar os edifícios de uma quadra num único bloco (MENDONÇA et al., 2013; BASTIÁN-MONARCA; SUÁRES; ARENAS, 2016) ou ainda restringir o mapeamento acústico ao período diurno, (FIEDLER; ZANNIN, 2015), dada as dificuldades para a realização de medidas noturnas. Em outras situações as ruas são classificadas em categorias de acordo com a intensidade do fluxo de veículos, de forma a otimizar a quantidade de medidas necessárias para construção de mapas de ruído, especialmente, em áreas de estudo da dimensão de cidades (BASTIÁN-MONARCA, SUÁRES; ARENAS, 2016). O fluxo e a composição do tráfego veicular determinados a partir dos dados dos CEV, utilizados nesse estudo, vêm contornar essas dificuldades.

A Directiva 2002/49/EC estabelece $L_{d e n} e L_{n}$ como principais indicadores para avaliação e gestão do ruído ambiental. O parâmetro $L_{d e n}$ representa a exposição relativa ao ruído ambiental médio nas $24 \mathrm{~h}$ do dia (dia-entardecer-noite). É definido como o nível de pressão sonora equivalente e contínuo, ponderado A, relativo ao período diurno (6h$18 \mathrm{~h}$ ), entardecer (18h-22h) e noturno (22h-6h). Existe ainda a aplicação de penalidades diferenciadas para os NPS durante o período do entardecer (correção $+5 \mathrm{~dB}$ ) e o noturno (correção $+10 \mathrm{~dB}$ ). O parâmetro acústico $L_{n}$ foi projetado para avaliar a exposição noturna, sendo definido como o nível de pressão sonora equivalente e contínuo, ponderado A, referente a um período de $8 \mathrm{~h}$ avaliado entre $22 \mathrm{~h}$ e $6 \mathrm{~h}$ (EEA, 2014; DIRECTIVA, 2002). A equação 1 apresenta a definição do parâmetro $L_{d e n}$ :

$$
L_{\text {den }}=10 \cdot \log \left(\frac{12}{24} 10^{\frac{L_{\text {dia }}}{10}}+\frac{4}{24} 10^{\frac{L_{\text {ent }}+5}{10}}+\frac{8}{24} 10^{\frac{L_{n}+10}{10}}\right)
$$

onde $L_{\text {dia }}$ é o nível de pressão sonora equivalente e contínuo referente a um período corresponde a $12 \mathrm{~h}$ avaliado entre $6 \mathrm{~h}$ e $18 \mathrm{~h}$. $L_{\text {ent }}$ é o nível de pressão sonora equivalente e contínuo referente a um período corresponde a $4 \mathrm{~h}$ avaliado entre $18 \mathrm{~h}$ e $22 \mathrm{~h}$ (entardecer).

As penalizações incorporadas ao indicador $L_{d e n}$ o tornam mais representativo do que $L_{\text {dia }}$ e ao mesmo tempo a utilização do $L_{d e n}$ possibilita a avaliação do incômodo, através dos \%PI (Percentual de Pessoas Incomodadas) e \%PAI (Percentual de Pessoas Altamente Incomodadas), que podem ser calculados para os diferentes modos de transporte (EC, 2002). A estimativa do percentual da população exposta por faixa de NPS utilizando $L_{\text {den }}$ facilita, por exemplo, a comparação da exposição ao ruído ambiental em cidades europeias (ARANA; SAN MARTIN; SALINAS, 2014), onde o mesmo índice é utilizado.

Esse conjunto de indicadores é amplamente utilizado como métrica na avaliação do ruído ambiental tanto no Brasil (CARVALHO JR. et al., 2013; GARAVELLI et al., 2014; FIEDLER; ZANNIN, 2015) como na Europa (KING; MURPHY; RICE, 2011), na América do Norte (SEONG et al., 2011), na América do Sul (SUÁREZ; BARROS, 2014) e na Ásia (CAl et al., 2015).

A determinação da população exposta ao ruído está entre os principais objetivos da Directiva 2002/49/EC, sendo um dado fundamental para o planejamento de ações destinadas a mitigar o ruído. De acordo com estimativas recentes, 654 mil anos de vida 
saudáveis são perdidos todos os anos em áreas urbanas na Europa Ocidental devido ao incômodo com o ruído (LICITRA; ASCARI; BRAMBILLA, 2012).

A avaliação do incômodo provocado pelo ruído dos transportes é um dos maiores desafios para a comunidade acadêmica. Pesquisas realizadas desde a década de 70 (Schultz, 1978) avaliaram o incômodo provocado pelo transporte rodoviário, ferroviário e aeronáutico. Os resultados são apresentados na forma de equações dose-resposta que expressam de forma clara a relação entre dose (causa-ruído) e resposta (efeitoincômodo). Por meio destas equações estima-se o incômodo provocado pelo ruído em função da exposição. A Directiva 2002/49/EC recomenda como descritores o \% PI e/ou \%PAI.

Destaque deve ser dado à avaliação do incômodo do ruído noturno. Há evidências científicas que apontam os efeitos do ruído no sono e as relações entre sono e saúde (AMUNDSEN; KLÆBOE, 2013; HALPERIN, 2014; MÜNZEL et al., 2020). A partir do $L_{n}$, parâmetro acústico que representa os níveis de pressão sonora no período noturno, é possível determinar o \%PDS (Percentual de Pessoas com Distúrbios no Sono) e o \%PSAP (Percentual de Pessoas com Sono Altamente Perturbado) (MIEDEMA; PASSCHIERVERMEER; VOS, 2003; EC, 2004; WHO, 2018).

Observa-se ainda que as relações dose-resposta deverão ser utilizadas como método para avaliar o efeito do ruído sobre a saúde da população, sendo, conforme a Directiva 2002/49/EC $L_{\text {den }}$ o indicador de ruído associado ao incômodo (\%PI e \%PAl) e $L_{n}$ o indicador de ruído associado as perturbações do sono (\%PDS e \%PSAP).

As equações 2 e 3 são as aproximações polinomiais para a estimativa do \%PI e \%PAl com o ruído devido ao transporte rodoviário (EC, 2002).

$$
\begin{aligned}
& \% P I=1,795 \times 10^{-4}\left(L_{d e n}-37\right)^{3}+2,110 \times 10^{-2}\left(L_{d e n}-37\right)^{2}+0,5353\left(L_{d e n}-37\right) \\
& \% P A I=9,868 \times 10^{-4}\left(L_{d e n}-42\right)^{3}-1,436 \times 10^{-2}\left(L_{d e n}-42\right)^{2}+0,5118\left(L_{d e n}-42\right)
\end{aligned}
$$

A estimativa dos percentuais da população que apresenta distúrbios no sono (\%PDS) e com o sono altamente perturbado (\%PSAP) com o ruído do transporte rodoviário (EC, 2002) é feita por meio das equações 4 e 5.

$$
\begin{aligned}
& \% P D S=13,8-0,85 L_{n}+0,01670 L_{n}{ }^{2} \\
& \% P S A P=20,8-1,05 L_{n}+0,01486 L_{n}{ }^{2}
\end{aligned}
$$

\section{Método}

Brasília, capital federal do Brasil, é também a sede do governo do Distrito Federal. O plano urbanístico da capital, conhecido como Plano Piloto, foi elaborado por Lúcio Costa, que, aproveitando o relevo da região, adequou-o ao projeto do lago Paranoá, concebido em 1893 pela Missão Cruls (COSTA, 2011).

O Plano Piloto é delineado pelo Eixo Rodoviário no sentido norte-sul e pelo Eixo Monumental no sentido Leste-Oeste. O Eixo Rodoviário é formado pelas asas Sul e Norte e pela parte central, onde as asas se encontram sob a Rodoviária. As asas são áreas compostas basicamente pelas Superquadras residenciais, quadras comerciais e entre quadras de lazer e de diversão. O Eixo Monumental localizada no centro do Plano Piloto, faz a ligação entre a Rodoferroviária de Brasília (a oeste) e a Praça dos Três Poderes (a leste). Na Figura 1 é apresentado o mapa de Brasília, com a área de estudo.

Os dados do tráfego rodoviário, necessários para a geração dos mapas acústicos, foram coletados pelos CEV (controladores eletrônicos de velocidades) (MAROJA et al., 2011) e 
completados com medições e filmagens in situ. Além de aferir a velocidade dos veículos, os CEV registram a contagem do fluxo e a composição do tráfego veicular por hora, ao longo de todos os dias do ano, permitindo a avaliação detalhada do impacto ambiental inclusive no período noturno. Na modelagem foi considerada uma área com $69,2 \mathrm{~km}^{2}$, contorno vermelho destacado na Figura 1, maior que a área de interesse.

Figura 1 - Área de estudo e a localização dos CEV instalados em Brasília, pontos verdes

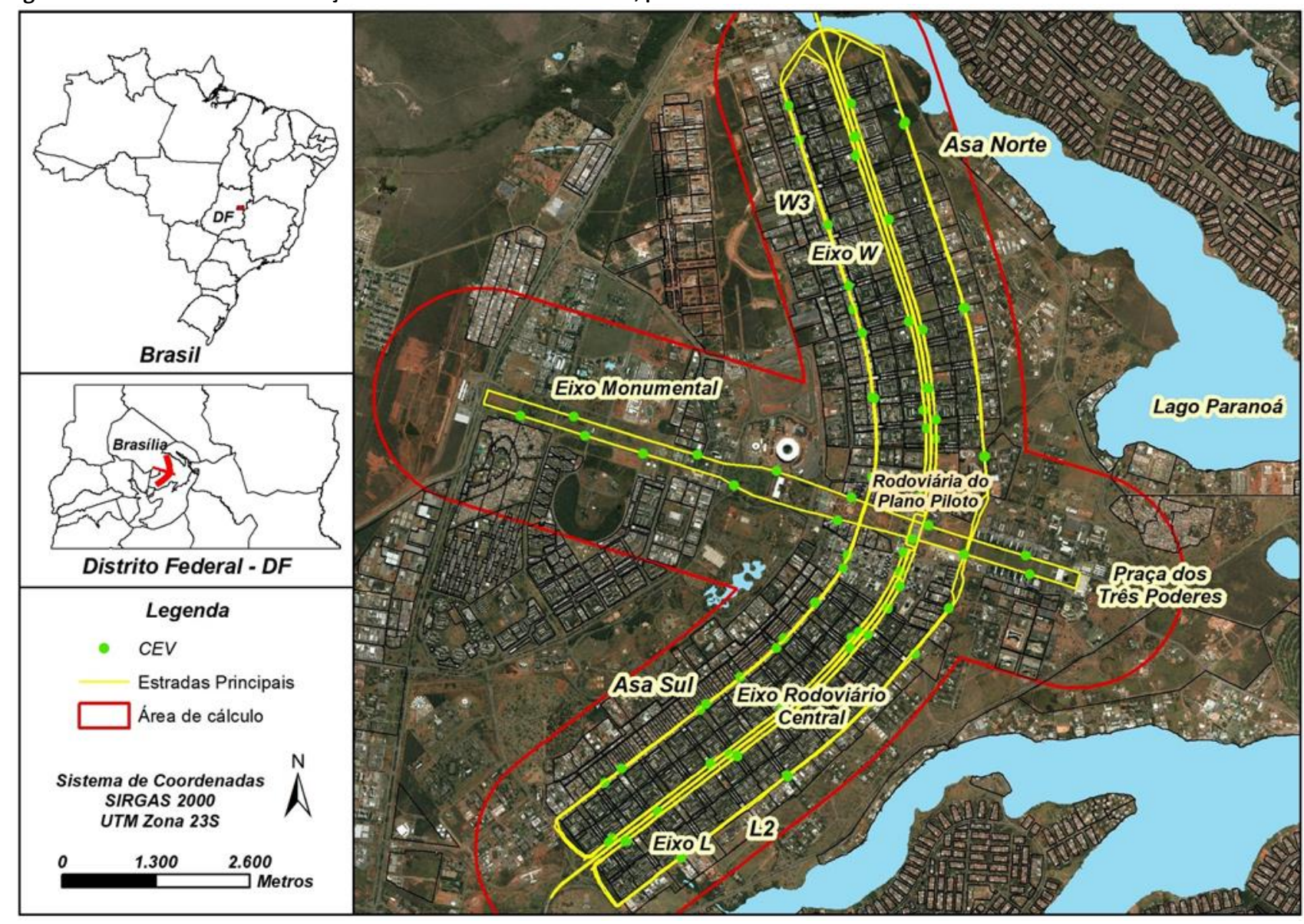

Fonte: os autores.

Os mapas produzidos foram combinados com dados demográficos do Instituto Brasileiro de Geografia e Estatística (IBGE), o que permitiu calcular a população e área exposta por faixa de ruído para os parâmetros $L_{d e n} e L_{n}$, além dos percentuais de pessoas incomodadas e com distúrbios no sono devido ao tráfego rodoviário.

Os dados relativos ao fluxo e a composição do tráfego das principais vias na área de estudo: Eixo Rodoviário Central; Eixo Monumental; Eixo L; Eixo W; Avenida W3 e Avenida L2, foram determinados a partir dos relatórios de tráfego dos $91 \mathrm{CEV}$, em destaque na Figura 1. Os dados foram fornecidos pelo Departamento de Trânsito do Distrito Federal (DETRAN-DF) e do Departamento de Estradas de Rodagem do (DERDF). No mapa da Figura 1, alguns aparelhos aparecem sobrepostos devido à escala. A partir dos relatórios, foi elaborado o perfil de fluxo médio de veículos por hora/categoria para cada pista de cada via da área de estudo.

O NPS em cada receptor foi calculado considerando efeitos de reflexão e da propagação da onda sonora, os mapas de contorno foram produzidos a partir de uma rede com 2.771.489 receptores.

O cálculo do mapa de ruído é realizado a partir de uma rede de receptores de ruído sobreposta à área de estudo considerada. A localização dos receptores e a precisão dos 
cálculos estabelecem as dimensões da rede, o passo define as dimensões de cada célula da rede. A altura de cálculo representa a posição dos receptores em relação ao solo e a resolução espacial tem relação com a precisão do mapa. O passo utilizado foi de $5 \mathrm{~m}$, adequado para produção de mapas de ruído com a dimensão de cidades. A altura de cálculo foi de $4 \mathrm{~m}$ e a resolução espacial foi a maior disponível, $9 \times 9(\mathrm{~m})$, no software SoundPLAN®, utilizado neste estudo.

A partir das curvas de nível do Sistema Cartográfico do DF-SICAD-2009, foi construído o MDT (Modelo Digital de Terreno) do mapa, depois inseridos os edifícios, casas e conjuntos comerciais com as respectivas alturas em relação ao MDT. Em seguida foram incorporadas as linhas de emissão sonora referentes às vias que atravessam a região de interesse, com a distribuição do fluxo de veículos por hora e composição do tráfego veicular característico de cada via. Seguindo a Directiva 2002/49/EC foi utilizado o modelo francês para a predição do ruído gerado pelo tráfego rodoviário NMPB-Routes1996.

A calibração dos mapas de ruídos tem o objetivo de verificar se os NPS previstos, por meio da simulação estão coerentes com as medidas realizadas em campo. A coleta de dados para a calibração foi realizada em cinco locais na área central e doze locais distribuídos igualmente na Asa Sul e Asa Norte. As medidas foram efetuadas no exterior das edificações a pelo menos 3,5 $\mathrm{m}$ de qualquer estrutura refletora, sendo o tempo de coleta $15 \mathrm{mim}$, com o sonômetro posicionado a uma altura de 4,0 m acima do solo. Foram realizadas em dias normais da semana, sem chuva e baixa velocidade do vento, no período das $7 \mathrm{~h}$ às $20 \mathrm{~h}$. Os pontos de medição foram selecionados de forma que o ruído predominante era o oriundo das vias, cada ponto foi georreferenciado com a utilização de GPS. Para as medições acústicas foi utilizado o sonômetro SOLO da $01 \mathrm{~dB}$, com tripé e protetor de vento, foi utilizada a curva de ponderação $A$, com filtro de frequência de $1 / 3$ de oitavas.

Para determinar a população exposta por faixa de NPS foram selecionados os dados do Censo Demográfico 2010, disponibilizados pelo IBGE (IBGE, 2011). A população por setor censitário na área de estudo foi associada espacialmente aos setores censitários utilizando o Sistema de Informações Geográfica (SIG) ArcGIS®. Os habitantes de cada setor censitário foram distribuídos pelos edifícios do setor, proporcionalmente ao número de andares e a área do edifício. Em seguida foi determinada a intersecção da fachada dos edifícios com as isofônicas de ruído relativas aos indicadores $L_{d e n} e L_{n}$.

A população exposta por classe dos indicadores foi calculada determinando para cada edifício a porção da fachada atingida por cada faixa de NPS utilizando o SIG ArcGIS®. Esses dados, substituídos nas equações de 2 a 5, foram utilizados para determinar os percentuais de pessoas incomodadas e as pessoas com distúrbios no sono, em função da exposição ao ruído gerado pelo tráfego rodoviário.

\section{Resultados e discussão}

A Tabela 1 apresentada os resultados simulados, Leq (S), medidos in situ, Leq (M) e a diferença (Dif) entre os dois valores. $X(\mathrm{~m})$ representa a latitude e $\mathrm{Y}(\mathrm{m})$ a longitude do local da medida, o sistema de coordenadas utilizado foi o SIRGAS 2000-UTM 23S. Na calibração dos mapas os receptores foram posicionados nos mesmos locais das medidas de campo e considerado o mesmo horário da medição.

As diferenças entre os valores simulados e os medidos apresentaram valores absolutos menores ou iguais a 3,0 dB e média de $1,5 \mathrm{~dB}$. O resultado é compatível com outras pesquisas (SUÁREZ; BARROS, 2014; CAI et al., 2015; FIEDLER; ZANNIN, 2015). Portanto, os resultados indicam que a metodologia proposta neste artigo, da utilização dos dados 
gARAVELLI, S. L.; MAROJA, A. de M.; CARVALHO JÚNIOR, E. B. de.; COSTA, C. A. da.

Exposição ao ruído do transporte rodoviário em Brasília, Brasil

dos CEV para a elaboração de mapas acústicos é adequada e no caso os mapas elaborados possuem precisão de 3,0 dB, no mínimo.

Tabela 1 - Calibração do mapa de ruído - comparação entre valores medidos e simulados

\begin{tabular}{|c|c|c|c|c|c|c|c|}
\hline & Ponto & Local & $X(m)$ & $Y(m)$ & Leq(S) & Leq(M) & Dif. \\
\hline \multirow{5}{*}{$\begin{array}{c}\text { Área } \\
\text { central }\end{array}$} & 1 & Praça dos Três Poderes & 193444,92 & 8251044,90 & 62,8 & 61,1 & 1,7 \\
\hline & 2 & Câmara dos Deputados & 192819,51 & 8251233,27 & 60,5 & 58,8 & 1,7 \\
\hline & 3 & Torre de TV & 190077,19 & 8252085,44 & 59,4 & 59,4 & 0,0 \\
\hline & 4 & Memorial JK & 187881,51 & 8252768,59 & 64,7 & 62,3 & 2,4 \\
\hline & 5 & Nossa Senhora da Paz & 186532,02 & 8253174,13 & 61,1 & 61,8 & $-0,7$ \\
\hline \multirow{6}{*}{ Asa Sul } & 1 & Eixo Central Sul & 190140,79 & 8249973,67 & 74,9 & 73,9 & 1,0 \\
\hline & 2 & Eixo Central Sul & 188483,44 & 8248557,12 & 74,3 & 73,9 & 0,3 \\
\hline & 3 & Eixo L Sul & 189571,17 & 8249593,57 & 73,2 & 70,3 & 2,9 \\
\hline & 4 & L2 Sul & 190620,90 & 8249500,34 & 68,1 & 65,1 & 3,0 \\
\hline & 5 & W3 Sul & 188119,27 & 8249259,68 & 72,9 & 72,2 & 0,7 \\
\hline & 6 & W3 Sul & 189208,71 & 8250173,10 & 75,5 & 72,6 & 2,9 \\
\hline \multirow{6}{*}{ Asa Norte } & 1 & Eixo Central/Eixo W & 190439,75 & 8256718,49 & 73,2 & 71,1 & $-2,1$ \\
\hline & 2 & Eixo Central/Eixo L & 190511,88 & 8256751,06 & 72,1 & 69,8 & $-2,3$ \\
\hline & 3 & 711 Norte & 189833,90 & 8256089,93 & 67,8 & 66,9 & $-0,9$ \\
\hline & 4 & 609/610 Norte & 191469,13 & 8256079,02 & 61,3 & 63,5 & 2,2 \\
\hline & 5 & 611 Norte & 191271,97 & 8256793,14 & 53,4 & 53,3 & $-0,1$ \\
\hline & 6 & 412/413 Norte & 190975,89 & 8257056,12 & 51,3 & 52,6 & 1,3 \\
\hline
\end{tabular}

Fonte: os autores.

As Figuras 2 e 3 apresentam os mapas referentes aos indicadores $L_{d e n}$ e $L_{n}$. A área de cálculo apresentada nas figuras corresponde a uma faixa de $3 \mathrm{~km}$ centrada com o eixo rodoviário na direção Norte-Sul e com o eixo monumental ao longo da direção LesteOeste, correspondendo a uma área total de 69,2km2.

Figura 2 - Mapa de ruído período dia $(24 h)\left(L_{d e n}\right)$
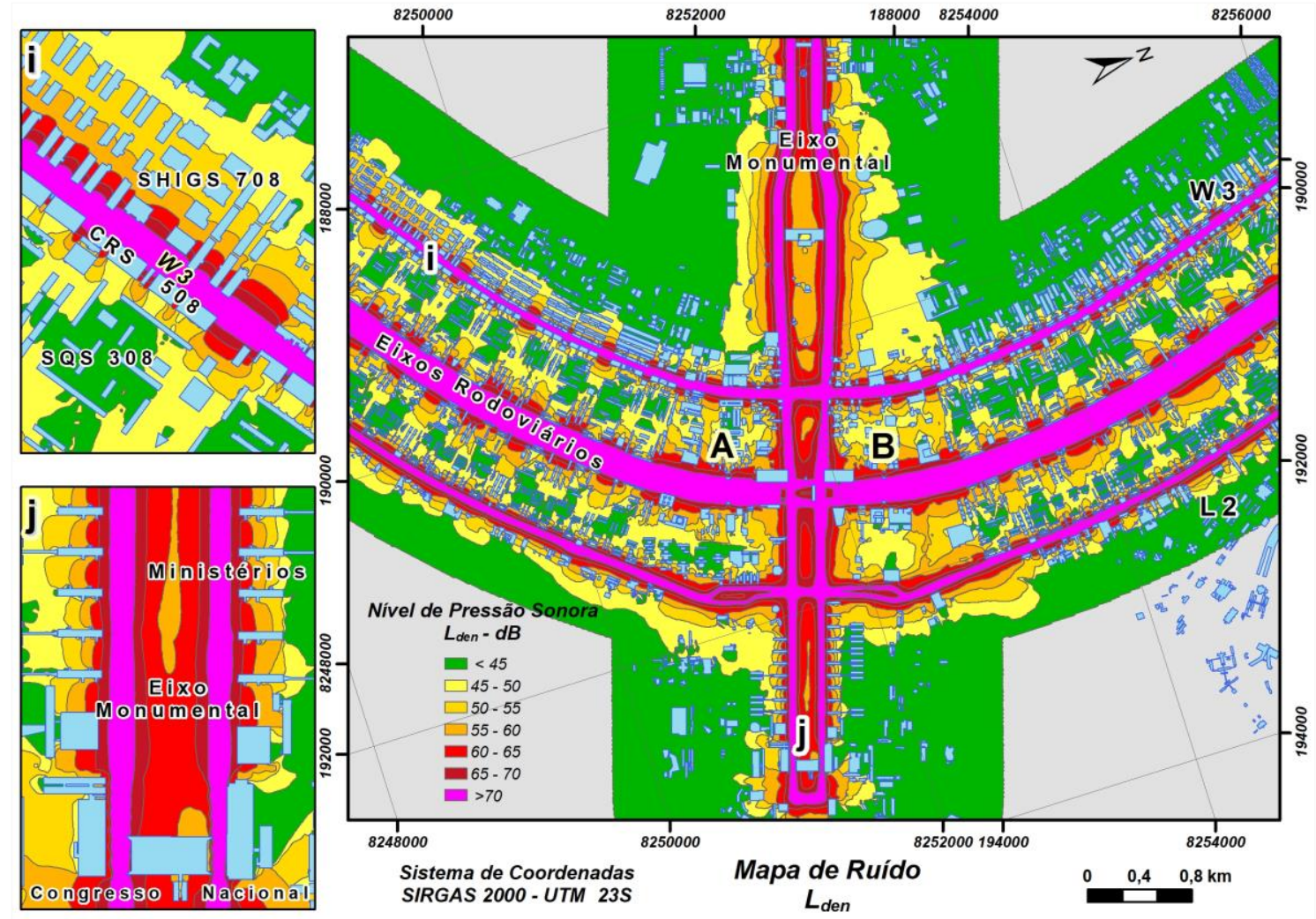

Fonte: os autores. 


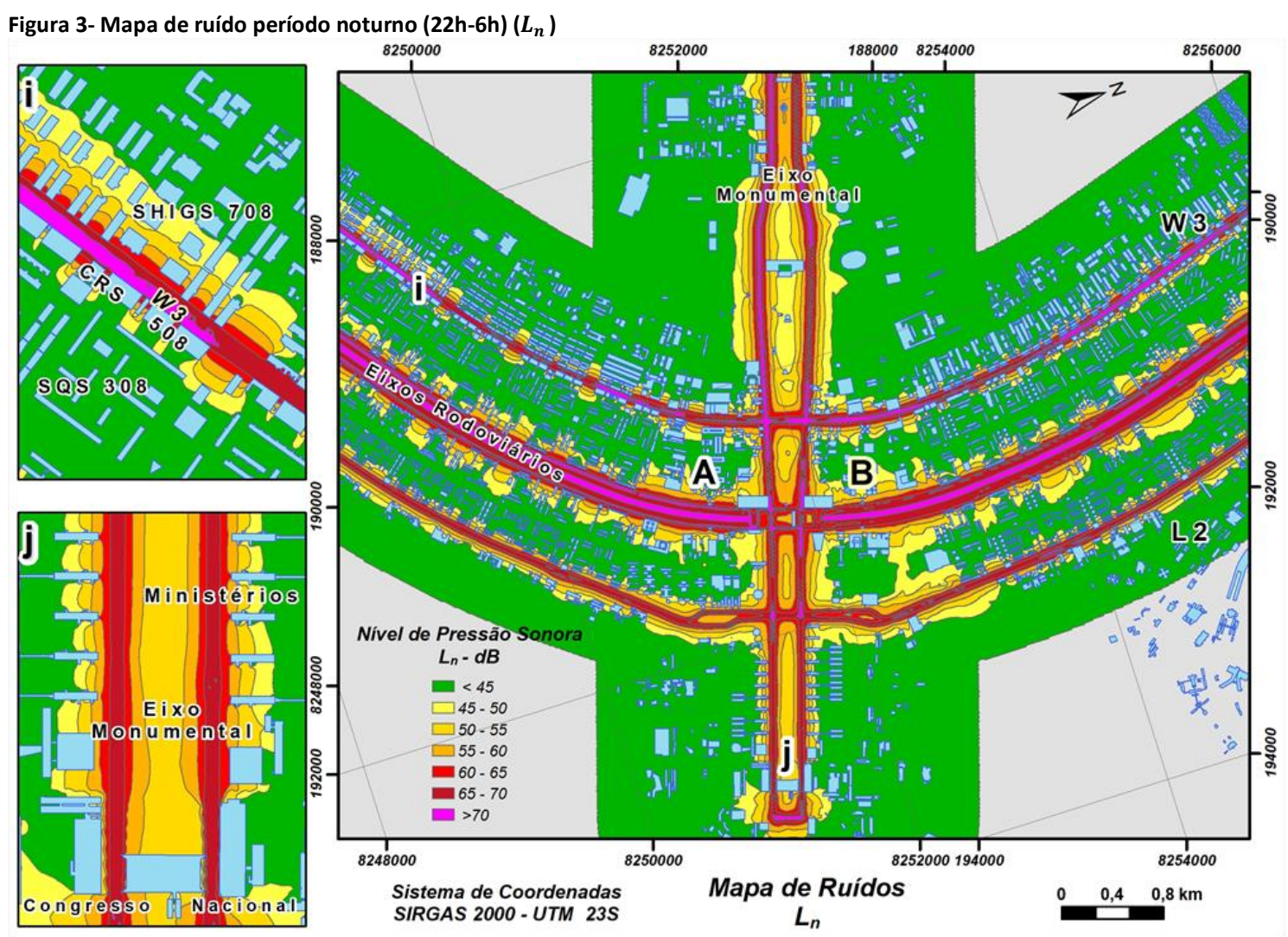

Fonte: os autores.

Nas Figuras 2 e 3, a esquerda de cada mapa é apresentada a ampliação de duas regiões do mapa principal, em (i) a região próxima a Superquadra SQS 308 da Asa Sul, considerada a Superquadra modelo de Brasília (MACHADO, 2007). Em (j) está a Esplanada dos Ministérios, onde se encontram o Palácio da Alvorada, o Supremo Tribunal Federal, o Congresso Nacional e os Ministérios. As faixas em rosa, NPS > 70dB, identificam o eixo das vias na Figura 1. Na Figura 2 observa-se que as faixas em rosa foram substituídas por tons de vermelho e vermelho escuro (NPS > $60 \mathrm{~dB}$ ). Em (i) essas faixas representam a Avenida W3 e em (j) o Eixo Monumental.

No mapa principal as faixas coloridas em cores mais fortes identificam o conjunto das vias, apresentadas na Figura 1. A comparação entre as Figuras 2 e 3 evidencia que no período noturno os NPS estão pelo menos $5 \mathrm{~dB}$ abaixo dos valores observados no período diurno.

Referente ao tráfego rodoviário, o Guideline Development Group (GDG) recomenda níveis de ruído máximos de $53 \mathrm{~dB}$ para $L_{d e n}$. Já para exposição ao ruído noturno recomenda o limite de $45 \mathrm{~dB}$ para $L_{n}$. O GDG considera que exposições a níveis de ruído acima destes limites estão associadas a efeitos adversos à saúde (WHO, 2018).

Os resultados mostram que o ruído provocado pelo tráfego rodoviário na fachada dos edifícios residenciais localizados às margens das principais vias encontra-se mais de 15 $\mathrm{dB}$ acima dos limites recomendados pelo GDG. Mesmo na região administrativa da Esplanada dos Ministérios os NPS na fachada dos Ministérios e do Congresso Nacional ultrapassam $10 \mathrm{~dB}$ os limites indicados acima.

Em (i) e em (j), Figuras 2 e 3, observa-se claramente a diferença dos NPS entre regiões onde os prédios são paralelos e perpendiculares a via. Observa-se que nas margens da Avenida $W_{3}$ as regiões de conformidade estão mais presentes nas quadras 300 , devido 
gARAVElli, S. L.; MAROJA, A. de M.; CARVALHO JÚNIOR, E. B. de.; COSTA, C. A. da.

Exposição ao ruído do transporte rodoviário em Brasília, Brasil

ao posicionamento dos edifícios e ao fato das quadras comerciais 500 servirem como barreira acústica.

Os NPS elevados observados nas regiões A e B destacadas nos mapas apresentados nas Figuras 2 e 3, região central da cidade, devem-se a convergência dos eixos rodoviários e do eixo monumental. Observa-se a predominância dos tons de laranja a rosa, indicando ruído ambiental elevado quando comparado às demais regiões da cidade. Apesar da região ser predominantemente administrativa, comercial e de diversão, abriga também nas margens do eixo leste e do eixo oeste os principais hospitais da cidade devendo ser uma das primeiras regiões com atenção em medidas de mitigação em relação ao ruído ambiental.

A Figura 4 apresenta os NPS nas fachadas dos prédios em duas das regiões em destaque mostradas nas Figuras 2 e 3. Os moradores de edifícios nas margens das vias convivem com NPS elevados, $L_{\text {den }}$ acima de $70 \mathrm{~dB}$, valores superiores ao limite conforme WHO (2018). Na Esplanada dos Ministérios também são observados NPS elevados nas fachadas mais próximas do eixo monumental.
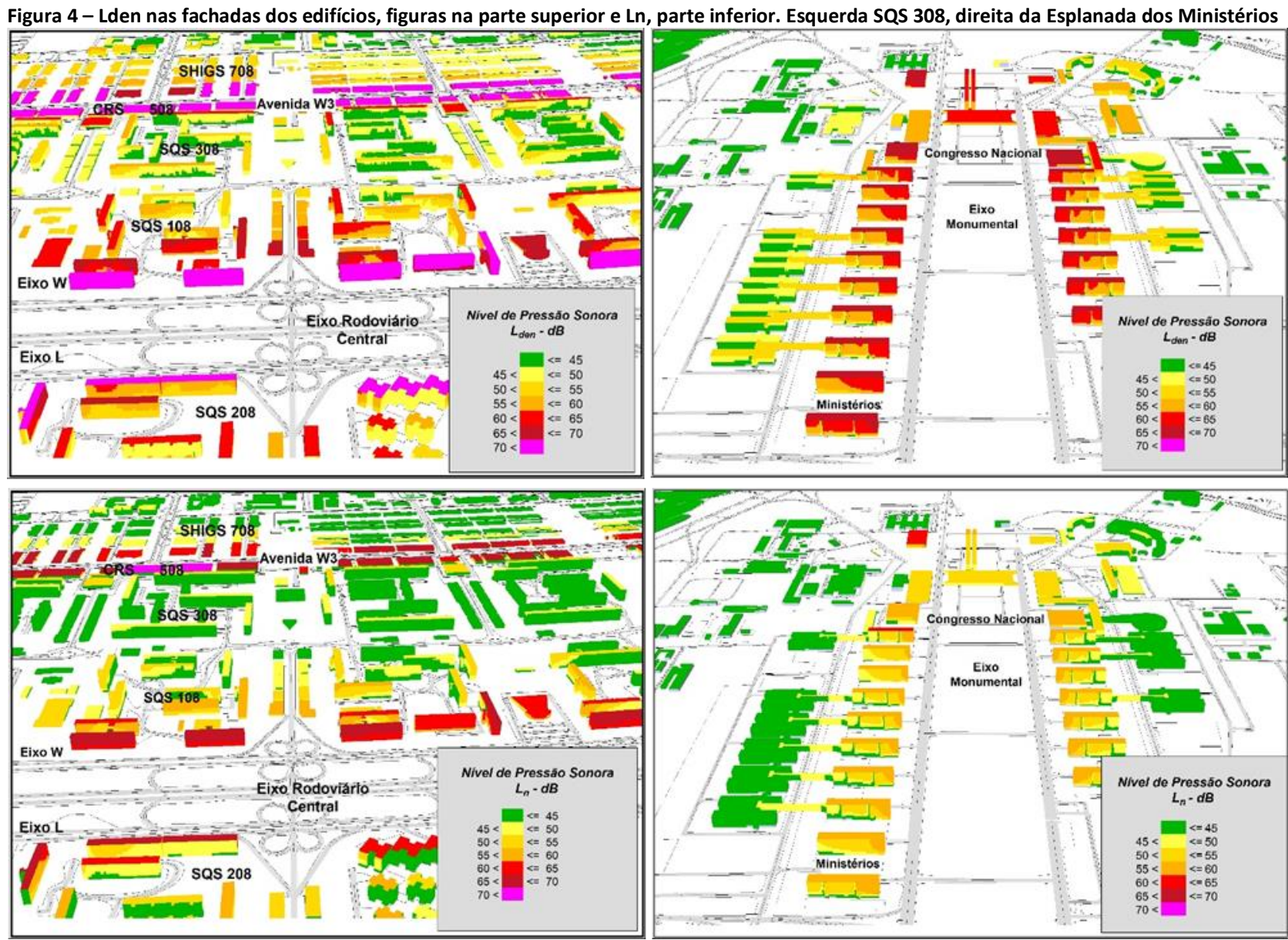

Fonte: os autores.

Nos mapas acústicos, pode-se notar que os edifícios mais próximos das vias principais funcionam como barreiras acústicas, levando a um decaimento significativo do ruído que se propaga para os edifícios localizados na parte interna da quadra residencial.

A Tabela 2 apresenta os resultados do número de pessoas e percentual da população exposta por classe dos indicadores $L_{d e n}$ e $L_{n}$. As estatísticas da Tabela 2 foram elaboradas considerando a população da área de estudo de 227.894 habitantes. 
gARAVELLI, S. L.; MAROJA, A. de M.; CARVALHO JÚNIOR, E. B. de.; COSTA, C. A. da.

Exposição ao ruído do transporte rodoviário em Brasília, Brasil

\begin{tabular}{|c|c|c|c|c|c|c|c|}
\hline & $\leq 50$ & $50-55$ & $55-60$ & $60-65$ & $65-70$ & $\geq 70$ & $\Sigma>55$ \\
\hline \multirow{2}{*}{$L_{\text {den }}$} & 138.252 & 47.851 & 17.882 & 11.428 & 7.772 & 4.709 & 41.791 \\
\hline & $60,7 \%$ & $21,0 \%$ & $7,8 \%$ & $5,0 \%$ & $3,4 \%$ & $2,1 \%$ & $18,3 \%$ \\
\hline \multirow[t]{2}{*}{$L_{n}$} & 188.836 & 20.451 & 11.164 & 4.281 & 2.668 & 494 & 18.607 \\
\hline & $82,9 \%$ & $9,0 \%$ & $4,9 \%$ & $1,9 \%$ & $1,2 \%$ & $0,2 \%$ & $8,2 \%$ \\
\hline
\end{tabular}

Fonte: os autores.

Apesar do grande número de veículos que trafegam nas vias, o número de residências e prédios comerciais e administrativos afetados diretamente pelo ruído gerado é relativamente pequeno. Na região de estudo, a área central é essencialmente administrativa, assim o ruído gerado a partir dessas vias atinge um pequeno número de residentes. Outro fator que pode ser utilizado como justificativa para a pequena população exposta na faixa de ruído mais significativa (Tabela 2), é a distância relativa entre a via e as residências, em média $150 \mathrm{~m}$. Projetar vias com potencial de fluxo elevado, distantes de residências, é uma boa prática para a melhoria do clima acústico de uma cidade.

Conforme pode ser observado na Figura 2, as áreas expostas a NPS acima de $65 \mathrm{~dB}$ na região central de Brasília, são as faixas de rolamentos das vias $\mathrm{S} 1$ e N1 e suas vizinhanças. Assim os edifícios comerciais e administrativos localizados próximos às essas vias sofrem o impacto do ruído gerado pelo tráfego rodoviário, se enquadram nesta categoria o Setor Hoteleiro Norte e Sul e o Palácio do Buriti, sede do governo local. Aproximadamente $19 \%(13,1 \mathrm{~km} 2)$ da área de estudo encontra-se nessa situação. Para estas localidades recomenda-se atenção especial na elaboração do PRR (Plano de Redução de Ruídos).

A região situada na faixa entre 60 e $65 \mathrm{~dB}$, para o $L_{\text {den }}$, corresponde a $11 \%(6,5 \mathrm{~km} 2) \mathrm{da}$ área de estudo. A maior parte da área, $70 \%(48,7 \mathrm{~km} 2)$, encontra-se numa faixa dos NPS abaixo de $60 \mathrm{~dB}$.

Apesar do elevado fluxo de veículos observado nas vias do estudo, Eixo Central, Eixos $L$ e W, W3 e L2, o número de residências e prédios comerciais e administrativos afetado diretamente pelo ruído é relativamente pequeno. O percentual de residentes na faixa do $L_{d e n}$, maior que $70 \mathrm{~dB}$, é aproximadamente $2 \%$, sendo que para $L_{n}$ a quantidade é desprezível, conforme pode ser observado na Tabela 2.

Observam-se que $8,4 \%$ e 3,1\% da população estudada estão expostas, respectivamente, a valores de $L_{d e n}$ e $L_{n}$ entre 60 e $70 \mathrm{~dB}$. Esses moradores estão localizados nos edifícios mais próximos aos Eixos $L$ e W, além das vias W3 e L2. Conforme mostram os mapas de ruído, em muitos casos estes edifícios funcionam como barreiras acústicas dificultando a propagação do ruído para o interior das quadras.

A Tabela 2 indica ainda que $18,3 \%$ da população está exposta ao $L_{\text {den }}$ superior a $55 \mathrm{~dB}$, limite superior estabelecido pela WHO (2018), indicando a necessidade da implantação do Plano de Redução de Ruídos (PRR). Os NPS nas vias Eixo Central, Eixos L e W, W3 e L2 apresentam um potencial de impacto significativo na população residente próximo às mesmas, o que não ocorre para os habitantes do interior das quadras.

Na Tabela 3 são apresentados os resultados da estimativa do número de pessoas incomodadas e altamente incomodadas pelo tráfego rodoviário em função do $L_{d e n}$. Os cálculos foram realizados utilizando as equações 2 e 3, onde foram substituídos o valor médio do parâmetro. Por exemplo, para a faixa $50 \leq L_{\text {den }}<55$, foi utilizado o valor de 52,5. Na segunda coluna é apresentada a população exposta por faixa do indicador $L_{d e n}$, com o respectivo percentual. Na última linha é apresentada a estimativa do número de pessoas incomodadas e altamente incomodadas, assim como o percentual em relação a população da área de estudo de 227.894 habitantes. 
gARAVELli, S. L.; MAROJA, A. de M.; CARVALHO JÚNIOR, E. B. de.; COSTA, C. A. da.

Exposição ao ruído do transporte rodoviário em Brasília, Brasil

Tabela 3 - Estimativa do percentual de pessoas incomodadas e altamente incomodadas pelo tráfego rodoviário

\begin{tabular}{cccccc}
\hline & População exposta & \multicolumn{2}{c}{ Incomodados } & \multicolumn{2}{c}{ Altamente incomodados } \\
\cline { 3 - 6 } & & $\%$ & Pessoas & $\%$ & Pessoas \\
\cline { 3 - 6 } & $4.709(2,1 \%)$ & 53,6 & $2.525(1,1 \%)$ & 30,2 & $1.424(0,6 \%)$ \\
\hline$L_{\text {den }} \geq 70$ & $7.772(3,4 \%)$ & 41,0 & $3.190(1,4 \%)$ & 20,1 & $1.560(0,7 \%)$ \\
\hline $65 \leq L_{\text {den }}<70$ & $11.428(5,0 \%)$ & 30,3 & $3.468(1,5 \%)$ & 13,0 & $1.481(0,6 \%)$ \\
\hline $60 \leq L_{\text {den }}<65$ & $17.882(7,8 \%)$ & 21,4 & $3.824(2,7 \%)$ & 8,2 & $1.459(0,6 \%)$ \\
\hline $55 \leq L_{\text {den }}<60$ & $47.851(21,0 \%)$ & 14,0 & $6.716(2,9 \%)$ & 4,9 & $2.361(1,0 \%)$ \\
\hline $50 \leq L_{\text {den }}<55$ & \multicolumn{2}{c}{$19.724(8,7 \%)$} & & $8.285(3,6 \%)$ \\
\hline Totais & \multicolumn{5}{c}{}
\end{tabular}

Fonte: os autores.

Os resultados da Tabela 3 indicam que, apesar dos elevados NPS na vizinhança das vias principais, o rú́do advindo do tráfego rodoviário tem potencial de incomodar menos de 9\% da população residente em Brasília, sendo que os altamente incomodados não atinge 4\%. A configuração das Superquadras, onde se concentra a área residencial, propicia locais mais protegidos deste contaminante ambiental, contribuindo assim de maneira significativa para este fato. Pode-se observar também que os edifícios voltados para as vias principais funcionam como barreiras acústicas protegendo as áreas internas das quadras.

Na Tabela 4 são apresentados os resultados das pessoas sujeitas a distúrbios no sono e sujeitas a elevados distúrbios no sono provocados pelo tráfego rodoviário. Os cálculos foram realizados utilizando as equações 4 e 5 , onde foram substituídos o valor médio do parâmetro, por exemplo, para a faixa $50 \leq L_{n}<55$, foi utilizado o valor de 52,5 , mesmo procedimento utilizado na produção da Tabela 3. Na segunda coluna é apresentada a população exposta por faixa do indicador $L_{d e n}$, com o respectivo percentual. Na última linha é apresentada a estimativa do número de pessoas incomodadas e altamente incomodadas, assim como o percentual em relação a população da área de estudo de 227.894 habitantes.

Tabela 4 - Número e percentual de pessoas sujeitas a distúrbios e elevados distúrbios no sono

\begin{tabular}{cccccc}
\hline \multirow{2}{*}{$L_{n}$} & População exposta & \multicolumn{2}{c}{ Distúrbios no sono } & \multicolumn{2}{c}{ Elevados distúrbios no sono } \\
\cline { 3 - 6 } & & $\%$ & Pessoas & $\%$ & Pessoas \\
\cline { 3 - 6 } & $494(0,2 \%)$ & 40,0 & $197(0,1 \%)$ & 22,8 & $45(0,0 \%)$ \\
\hline $65 \leq L_{n}<70$ & $2.668(1,2 \%)$ & 32,5 & $867(0,4 \%)$ & 17,6 & $153(0,1 \%)$ \\
\hline $60 \leq L_{n}<65$ & $4.281(1,2 \%)$ & 25,9 & $1.109(0,5 \%)$ & 13,2 & $147(0,1 \%)$ \\
\hline $55 \leq L_{n}<60$ & $11.164(4,9 \%)$ & 20,1 & $2.248(1,0 \%)$ & 9,6 & $215(0,1 \%)$ \\
\hline $50 \leq L_{n}<55$ & $20.451(9,0 \%)$ & 15,2 & $3.109(1,4 \%)$ & 6,6 & $206(0,1 \%)$ \\
\hline Totais & & \multicolumn{2}{c}{$7.532(3,3 \%)$} & $766(0,3 \%)$ \\
\hline
\end{tabular}

Fonte: os autores.

Boa parte do tráfego de Brasília deve-se ao movimento pendular, de manhã há um grande fluxo de veículos que se deslocam de outras regiões administrativas para Brasília, que retornam no final da tarde. No período noturno o fluxo de veículos diminui consideravelmente, fato que é corroborado pelos percentuais de pessoas expostas a NPS mais elevados no período, representado pelo parâmetro $L_{n}$ e pelos resultados da estimativa do distúrbio do sono, apresentados na Tabela 4.

\section{Conclusão}

Os resultados indicam que a metodologia proposta para a coleta de dados do tráfego rodoviário é adequada, ou seja, a margem de erro observada, menor que $3 \mathrm{~dB}$ está na faixa encontrada na literatura para mapas de ruído ambiental. A utilização dos CEV é um procedimento que pode ser utilizado para a elaboração de mapas acústicos com a redução significativa dos custos. 
Em Brasília, o transporte individual é muito utilizado, em detrimento do coletivo, o que provoca um aumento significativo da quantidade de veículos nas vias. Além dos congestionamentos, que já são comuns nos horários de pico, o crescimento acelerado da frota e a opção pelo transporte individual provocam um aumento da contaminação acústica. Porém, apesar dos elevados NPS observados próximos às vias, o percentual de pessoas incomodadas e altamente incomodadas e com distúrbios no sono é relativamente pequeno.

Não há evidências que a poluição sonora tenha sido levada em conta no projeto urbanístico de Brasília, porém, os resultados deste artigo mostram uma influência positiva da concepção urbanística no clima acústico da cidade.

As principais vias de circulação possuem uma estrutura hierárquica, com as vias de circulação rápida localizadas no centro, no caso do Eixo, e mais distante das residências. Nas quadras residenciais as vias são sinuosas, sem saída, impedindo o tráfego de passagem e são de baixa velocidade, desestimulando o fluxo de veículos que não se destine a elas. A configuração das Superquadras propicia melhores condições de conforto acústico, sendo um modelo que pode ser utilizado no planejamento de outras áreas residenciais.

No estudo foram identificadas áreas para as quais são indicadas medidas para reduzir a exposição ao ruído do tráfego rodoviário no período diurno.

\section{Agradecimentos}

Os autores agradecem ao Conselho Nacional de Desenvolvimento Científico e Tecnológico-CNPq, a Universidade de Brasília UnB, ao Centro Universitário de Brasília - CEUB, a UNESCO e ao IBRAM pelos recursos financeiros aplicados no desenvolvimento do projeto e aos setores de geoprocessamento do Departamento de Trânsito do Distrito Federal-DETRAN-DF e do Departamento de Estradas de Rodagem do DF, DER-DF pelos dados do fluxo e composição do tráfego veicular. A Wesley C. de Melo e Edwin de S. Silva, pela colaboração nas medidas de campo.

\section{Referências}

AMUNDSEN, A. H.; KLÆBOE, R. Long-term effects of noise reduction measures on noise annoyance and sleep disturbance: The Norwegian façade insulation study. J. Acoust. Soc. Am., v.133, n.6, p. 3921-3928, jun. 2013. DOI: https://doi.org/10.1121/1.4802824.

ARANA, M.; SAN MARTIN, R.; SALINAS, J. C. People exposed to traffic noise in European agglomerations from noise maps. A critical review. Noise Mapp, v.1, p. 40-49, 2014. DOl: https://doi.org/10.2478/noise-2014-0005.

BASTIÁN-MONARCA, N. A.; SUÁREZ, E.; ARENAS, J.P. Assessment of methods for simplified traffic noise mapping of small cities: Casework of the city of Valdivia, Chile. Sci. Total Environ., v. 550, p. 439-448, 2016. DOI: http://dx.doi.org/10.1016/j.scitotenv.2016.01.139.

BUNN, F.; ZANNIN, P. H. T. Assessment of railway noise in an urban setting. Applied Acoustics, Paraná v. 104, p.1623, 25 outubro 2015. DOI: http://dx.doi.org/10.1016/j.apacoust.2015.10.025.

CAI, M.; ZOU, J.; XIE, J.; MA, X. Road traffic noise mapping in Guangzhou using GIS and GPS. Applied Acoustics, v. 87, p. 94-102, 2015. DOI: http://dx.doi.org/10.1016/j.apacoust.2014.06.005.

CARVALHO Jr., E. B.; GARAVELLI, S. L.; SMOZINSKI, F. V.; MAROJA, A. M.; MELO, W. C. Análise das principais métricas utilizadas no zoneamento acústico de áreas próximas a aeródromos. Journal of Transport Literature, v.7 n. 4, p. 175-198, out. 2013. DOI: https://doi.org/10.1590/\$2238-10312013000400009. 
CODEPLAN - COMPANHIA DE PLANEJAMENTO DO DISTRITO FEDERAL. PDAD Pesquisa Distrital por Amostra de Domicílios 2018 Distrito Federal, Brasília: CODEPLAN, 2018. Disponível em: http://www.codeplan.df.gov.br/wpcontent/uploads/2020/06/relatorio_DF_grupos_de_renda.pdf. Acesso em: 3 set. 2021.

COSTA, G. G. BRASÍLIA 50 ANOS: A Importância da Cartografia na Evolução Urbana do Distrito Federal. In: SIMPÓSIO DE CARTOGRAFIA HISTÓRICA,1., 2011, Paraty. Anais [...]. Belo Horizonte, CRCH-UFMG. Disponível em: https://www.ufmg.br/rededemuseus/crch/simposio/COSTA_GRACIETE_G_2.pdf. Acesso em: 01 de fevereiro de 2014.

D'ALESSANDRO, F.; SCHIAVONI, S. A review and comparative analysis of European priority indices for noise action plans. Sci. Total Environ., v. 518-519, p. 290-301, 2015. DOI: https://doi.org/10.1016/j.scitotenv.2015.02.102

DE VOS, P.; VAN BEEK. A. Environmental Noise. Encyclopedia of Environmental Health, p. 476-488, 2011. DOI: https://doi.org/10.1016/B978-0-444-52272-6.00252-X

DIRECTIVA 2002/49/CE do parlamento europeu e do conselho, relativa à avaliação e gestão do ruído ambiente. Jornal Oficial das Comunidades Europeias, v. L189, p. 12-25, 2002. Disponível em: https://eur-lex.europa.eu/legalcontent/PT/TXT/PDF/?uri=CELEX:32002L0049\&from=PT. Acesso em: 05 mar. 2012.

DISTRITO FEDERAL. Lei Distrital n. 4.092, de 30 de janeiro de 2008. Dispõe sobre o controle da poluição sonora e os limites máximos de intensidade da emissão de sons e ruídos resultantes de atividades urbanas e rurais. Diário Oficial do Distrito Federal, Distrito Federal, 01 fev. 2008. Disponível em:

http://www.sinj.df.gov.br/sinj/Norma/57055/Lei_4092_30_01_2008.html. Acesso em: 08 set. 2021.

EC- EUROPEAN COMMISSION. Position paper on dose-response relationships between transportation noise and annoyance. Luxembourg: Office for Official Publications of the European Communities, 2002. Disponível em: http://www.noiseineu.eu/en/2928-a/homeindex/file?objectid=2705\&objecttypeid=0. Acesso em: 13 set. 2021.

EC - EUROPEAN COMMISSION. Position paper on dose-effect relationships for night time noise. Working Group on Health and Socio-Economic Aspects, 2004. Disponível em: https://rigolett.home.xs4all.nl/Archiefgeluid/positionpaper-Lnight-11-11-2004.pdf. Acesso em: 13 set. 2021.

EEA - EUROPEAN ENVIRONMENTAL AGENCY, 2014. Noise in Europe 2014. Luxembourg: Publications Office of the European Union, 2014.Disponível em: https://www.eea.europa.eu/publications/noise-in-europe2014/at_download/FILE. Acesso em: 13 set. 2021.

FAJERSZTAJN, L.; GUIMARÃES, M. T.; DUIMD, E.; SILVA, T. G. V.; OKAMURAD, M. N.; BRANDÃO, S. L. B.; RIBEIRO, A. E.; NAUD, L. M.; O'SULLIVANC, S.; SALDIVA, P. H. N.; CARDOSO, M. R. A. Health effects of pollution on the residential population near a Brazilian airport: A perspective based on literature review. Journal of Transport \& Health, v. 14, p. 1-11, 2019. DOI: https://doi.org/10.1016/j.jth.2019.05.004

FIEDLER, P. E. K., ZANNIN, P. H. T. Evaluation of noise pollution in urban traffic hubs-Noise maps and measurements. Environmental Impact Assessment Review, v.51, p. 1-9, 2015. DOI:

http://dx.doi.org/10.1016/j.eiar.2014.09.014

GARAVELLI, S. L., MAROJA, A. M., COSTA, C. A., CARVALHO Jr., E. B. O projeto de Lúcio Costa para Brasília (DF) e a contaminação acústica. In: CONGRESSO LUSO BRASILEIRO PARA O PLANEJAMENTO URBANO, REGIONAL, INTEGRADO E SUSTENTÁVEL, 6, Lisboa, 2014. Livros de Actas [...]. Lisboa: PLURIS, 2014, p. 1664-1675.

GUEDES, I. C. M.; BERTOLI, S. R. Mapa acústico como ferramenta de avaliação de ruído de tráfego veicular em Aracaju - Brasil. PARC Pesquisa em Arquitetura e Construção, Campinas, v. 5, n. 2, p. 40-51, jul./dez. 2014. DOI: https://doi.org/10.20396/parc.v5i2.8634537

HALPERIN, D. Environmental noise and sleep disturbances: A threat to health? Sleep Science, v. 7, p. 209 - 212, 2014. DOI: https://doi.org/10.1016/j.slsci.2014.11.003 
IBGE - Instituto Brasileiro de Geografia e Estatística, 2011. Sinopse do Censo Demográfico 2010. 2011. Disponível em: https://www.ibge.gov.br/estatisticas/multidominio/genero/9662-censo-demografico-2010.html?=\&t=downloads.

Acesso em: 13 de setembro de 2021.

IPHAN - INSTITUTO DO PATRIMÔNIO HISTÓRICO E ARTÍSTICO NACIONAL (Brasil). Superintendência do Iphan no Distrito Federal. Superquadra de Brasília: preservando um lugar de viver, Brasília-DF, 2015. Disponível em: http://portal.iphan.gov.br/uploads/ckfinder/arquivos/cartilha_unidade_vizinhança_iphan_df.pdf. Acesso em: 05 set. 2018.

KEPHALOPOULOS, S.; PAVIOTTI, M.; ANFOSSO-LÉDÉE, F.; MAERCKE, D.V.; SHILTON, S.; JONES, N. Advances in the development of common noise assessment methods in Europe: The CNOSSOS-EU framework for strategic environmental noise mapping. Sci. Total Environ., n. 482-483, p. 400-410, 2014. DOI:

https://doi.org/10.1016/j.scitotenv.2014.02.031

KING, E.A.; MURPHY E.; RICE H.J. Implementation of the EU environmental noise directive: Lessons from the first phase of strategic noise mapping and action planning in Ireland. Journal of Environmental Management, v. 92, p. 756-764, 2011. DOI: https://doi.org/10.1016/j.jenvman.2010.10.034

KO, J.H.; CHANG, S.I.; KIM, M.; HOLT, J.B.; SEONG, J.C. Transportation noise and exposed population of an urban area in the Republic of Korea. Environment International, v. 37, p. 328-334, 2011. DOI:

https://doi.org/10.1016/j.envint.2010.10.001

LICITRA, G.; ASCARI, E.; BRAMBILLA, G. Comparative analysis of methods to estimate urban noise exposure of inhabitants. Acta Acustica united with Acustica, v. 98, p. 659 - 666, 2012. DOI: https://doi.org/10.3813/AAA.918546

MACHADO, M. P. Superquadra: pensamento e prática urbanística. 269 f, 2007. Dissertação (Mestrado em Arquitetura e Urbanismo) - Universidade de Brasília, Brasília, 2007.

MAROJA, A. M.; GARAVELLI, S. L.; CARVALHO JR, E. B. Noise mapping using vehicles electronic speed controllers. In: INTERNATIONAL CONGRESS ON SOUND AND VIBRATION, 18. Rio de Janeiro, 2011. Proceedings [... ]. Rio de Janeiro: ICSV, 2011.

MENDONÇA, A. B. D.; SURIANO, M. T.; SOUZA, L. C. L.; VIVIANI, E. Classes de quadras urbanas determinadas pelos níveis de ruídos. Revista Brasileira de Gestão Urbana (URBE), v.5, n. 2, p. 63-77, 2013. DOI:

https://doi.org/10.7213/urbe.05.002.SE05

MIEDEMA, H. M. E.; PASSCHIER-VERMEER, W.; VOS, H. Elements for a position paper on night-time transportation noise and sleep disturbance. TNO Inro report 2002-59. Disponível em:

https://d3pcsg2wjqgizr.cloudfront.net/files/o/articles/2792/noisesleepdisturbance.pdf. Acesso em: 13 de setembro de 2021.

MÜNZEL, T.; KRÖLLER-SCHÖN, S.; OELZE, M.; GORI, T.; SCHMIDT, F. P.; STEVEN, S.; HAHAD, O.; RÖÖSLI, M.; WUNDERLI, J. M.; DAIBER, A.; SØRENSEN, M. Adverse Cardiovascular Effects of Traffic Noise with a Focus on Nighttime Noise and the New WHO Noise Guidelines. Annu. Rev. Public Health, v.41, p. 29.1-29.20, 2020. DOI: https://doi.org/10.1146/annurev-publhealth-081519-062400

MURPHY, E., KING, E.A., RICE, H.J. Estimating human exposure to transport noise in central Dublin, Ireland. Environment International, v. 35, n.2, p.298-302,2009. DOI: https://doi.org/10.1016/j.envint.2008.07.026

PAIVA, K. M.; CARDOSO, M. R. A.; ZANNIN, P. H. T. Exposure to road traffic noise: Annoyance, perception and associated factors among Brazil's adult population. Science of the Total Environment, v. 650, p. 978-986, 2019. DOI: https://doi.org/10.1016/j.scitotenv.2018.09.041

SÃO PAULO (Município). Lei n. 16.499, de 20 de julho de 2016. Dispõe sobre a elaboração do Mapa do Ruído Urbano da Cidade de São Paulo e dá outras providências. Diário Oficial da cidade de São Paulo, São Paulo, 21 jul. 2016. 
gARAVELli, S. L.; MAROJA, A. de M.; CARVALHO JÚNIOR, E. B. de.; COSTA, C. A. da.

Exposição ao ruído do transporte rodoviário em Brasília, Brasil

SCHULTZ, T. J. Synthesis of social surveys on noise annoyance. Journal of the Acoustical Society of America, v. 64 , n. 2, p. 377 - 405, 1978. DOI: https://doi.org/10.1121/1.382013

SEO, K.; GOLUB, A.; KUBY, M. Combined impacts of highways and light rail transit on residential property values: a spatial hedonic price model for Phoenix, Arizona. Journal of Transport Geography, v. 41, p. 53-62, 2014. DOI: https://doi.org/10.1016/j.jtrangeo.2014.08.003

SEONG J. C.; PARK T.H.; KO, J. H.; CHANG, S. I.; KIM, M., HOLT, J. B.; MEHDI, M. R. Modeling of road traffic noise and estimated human exposure in Fulton County, Georgia, USA. Environment International, v. 37, p. 1336-1341., 2011. DOI: https://doi.org/10.1016/j.envint.2011.05.019

SØRENSEN, M.; HJORTEBJERG, D; ERIKSEN, K.T.; KETZEL, M.; TJØNNELAND, A. Exposure to long-term air pollution and road traffic noise in relation to cholesterol: A cross-sectional study. Environment International, v. 85, p. 238243, 2015. DOI: http://dx.doi.org/10.1016/j.envint.2015.09.021

SUÁREZ, E., BARROS, J. L. Traffic noise mapping of the city of Santiago de Chile. Sci. Total Environ, v. 466-467, p. 539-546, 2014. DOI: http://dx.doi.org/10.1016/j.scitotenv.2013.07.013

TSAI, K., LIN, M. Noise mapping in urban environments: a Taiwan study. Applied Acoustics, v. 70, p. 960-972, 2009. DOI: https://doi.org/10.1016/j.apacoust.2008.11.001

WHO - WORLD HEALTH ORGANIZATION, Environmental Noise Guidelines for the European Region, WHO Regional Office for Europe, Copenhagen, Denmark, 2018. Disponível em:

http://www.euro.who.int/en/publications/abstracts/environmental-noise-guidelines-for-the-european-region-2018. Acesso em: 05 set. 2020.

\section{Sérgio Luiz Garavelli}

Físico. Doutor em Física. Professor Titular no Centro Universitário de Brasília - CEUB. Endereço postal: SEPN 707/907 - Campus Universitário - Asa Norte, Brasília - Distrito Federal - Brasil, CEP: 70790-075.

\section{Armando de Mendonça Maroja}

Físico. Doutor em Física. Professor na Universidade de Brasília - Campus UnB de Planaltina. Endereço postal: Área Universitária n 1. Bairro: Vila Nossa Senhora de Fátima, Planaltina, Distrito Federal, Brasil. CEP: 73345-010.

\section{Edson Benício de Carvalho Júnior}

Engenheiro Civil. Doutor em Transportes. Professor na Universidade Católica de Brasília. Endereço postal: Universidade Católica de Brasília. QS 7 LOTE 1 - Taguatinga, Distrito Federal, Brasil, CEP: 71966-70.

\section{Cleber Alves da Costa}

Físico. Mestre em Planejamento e Gestão Ambiental. Endereço postal: Universidade Católica de Brasília. QS 7 LOTE 1 - Taguatinga, Brasília - DF, 71966-70 